\section{Cahiers de Narratologie}

Analyse et théorie narratives

Littérature et sciences

\title{
Le miroir, la cornue et la pierre de touche, ou : que peut la littérature pour la science?
}

Jean-Marc Lévy-Leblond

\section{(2) OpenEdition}

Journals

Édition électronique

URL : http://journals.openedition.org/narratologie/6002

DOI : 10.4000/narratologie.6002

ISSN : 1765-307X

Éditeur

LIRCES

Référence électronique

Jean-Marc Lévy-Leblond, « Le miroir, la cornue et la pierre de touche, ou : que peut la littérature pour la science ? », Cahiers de Narratologie [En ligne], 18 | 2010, mis en ligne le 15 juillet 2010, consulté le 19 avril 2019. URL : http://journals.openedition.org/narratologie/6002 ; DOI : 10.4000/narratologie.6002

Ce document a été généré automatiquement le 19 avril 2019

Article L.111-1 du Code de la propriété intellectuelle. 


\title{
Le miroir, la cornue et la pierre de touche, ou : que peut la littérature pour la science?
}

\author{
Jean-Marc Lévy-Leblond
}

\section{NOTE DE L'ÉDITEUR}

Ce texte a fait l'objet d'une publication antérieure dans La pierre de touche. La science à l'épreuve..., Paris, Gallimard Folio, 1996, p. 183-218. Nous remercions chaleureusement l'auteur - qui est intervenu dans le séminaire « Littérature et science(s) » en janvier 2009 - de nous avoir permis de le publier à nouveau dans ce numéro. Merci également à Serge Milan qui a œuvré techniquement pour que cette nouvelle publication soit possible.

1 La science, on ne le dira jamais assez, est jeune - en tout cas si l'on entend sous ce mot, "science", le mode présent de production des connaissances que nous appelons scientifiques, et pas seulement ces connaissances elles-mêmes. Car c'est bien la façon dont nous faisons la science, et ce que nous en faisons, qui nous pose tant de redoutables problèmes aujourd'hui. On ne saurait guère, pour remonter aux origines de cette science, de notre science, retourner en deçà du XVII ${ }^{\mathrm{e}}$ siècle et de sa révolution souvent appelée " galiléenne ", à si juste titre. La science est jeune, donc. Nouvelle venue dans le concert des arts et métiers, elle a besoin d'être éduquée, cultivée, policée. Encore adolescente, elle peut mal tourner. Elle a besoin de savoir qui elle est, de prendre une pleine conscience de sa nature propre, pour connaître ses limites et dompter ses tentations.

Dans cette " mise en culture ${ }^{1}$ », un rôle majeur revient, bien sûr, à la littérature : parce qu'elle apporte «ce qu'elle seule peut donner aux lecteurs: une connaissance approfondie, plus complexe, plus juste que celle qu'ils peuvent avoir par eux-mêmes de ce qu'ils sont, de ce qu'est leur condition, de ce qu'est leur vie ${ }^{2}$ ». En un temps où notre condition, notre vie sont soumises de plein fouet à l'impact de la technoscience, la 
littérature peut nous en donner une connaissance "plus complexe et plus juste » que beaucoup d'analyses théoriques, qu'elles soient historiques, épistémologiques ou sociologiques. Et cela vaut d'abord pour les scientifiques, dont l'enfermement dans leurs laboratoires ne facilite guère la prise de conscience. Ce qui suit peut ainsi être conçu comme une contribution à la mise en œuvre du "Connais-toi toi-même " socratique, à l'usage des scientifiques et - qui peut le plus, peut le moins ! - des profanes.

C'est donc délibérément que je m'intéresserai plus à ce que la littérature peut donner à la science qu'à ce qu'elle lui prend. Depuis longtemps, certes, la pratique littéraire a puisé dans l'activité scientifique nombre d'images, de métaphores, de modèles, de formes - et de nombreuses études ont été consacrées à ces emprunts. À titre d'exemple, chacun sait ce que Les affinités électives de Goethe doivent à la chimie; les bouleversements de la physique au début de ce siècle (relativité einsteinienne, théorie quantique) n'ont pas été sans influence sur des auteurs tels que Virginia Woolf, Faulkner ou Joyce ${ }^{3}$; quant aux mathématiques dites modernes et leur axiomatisation à la Bourbaki, les écrivains de l'Oulipo - Queneau, Roubaud, Perec, Calvino, etc. ont suffisamment explicité l'inspiration qu'ils y trouvaient ${ }^{4}$; Primo Levi a lui-même montré comment le chimiste en lui nourrissait l'écrivain ${ }^{5}$; et les références de Pynchon-à commencer par L'arc-en-ciel de la gravité - ne sont pas moins évidentes ${ }^{6}$. Mais outre qu'il serait fort présomptueux de ma part de m'avancer sur le terrain de la critique littéraire, je souhaite plutôt renverser le courant et montrer tout ce que, réciproquement, nous avons à gagner en examinant le spectre de la science au travers du prisme de la littérature... Ce petit guide de lecture, un simple carnet de notes, n'a évidemment aucune prétention à l'exhaustivité et ne fait qu'explorer quelques pistes à suivre. Pour un voyage autour de la science, voici donc quelques-uns des livres que je conseillerais d'emporter.

\section{Le miroir}

4 Et d'abord, demandons à la littérature de nous présenter la science, et ceux qui la font. Car la tour d'ivoire est pauvre en miroirs. Et les scientifiques ne connaissent guère leur image. Il est heureusement des livres où trouver ces reflets. Et si d'aucuns jugent ces miroirs déformants, c'est peut-être qu'ils ne sont que grossissants et mettent précisément en lumière les traits les plus caractéristiques de leurs objets - traits qui n'ont aucune raison d'être les plus flatteurs.

5 On sait le poids croissant que la science a exercé sur la culture au XIX ${ }^{e}$ siècle, et comment on lui a reproché d'étouffer la sensibilité, de désenchanter le monde, de trivialiser toute aspiration morale ou esthétique. Flaubert, plus que tout autre, témoigne de cette emprise, en même temps qu'il la met à distance. Rappelons-nous ainsi quelques articles du Dictionnaire des idées reçues:

«MATHÉMATIQUES : dessèchent le cœur.»

«SAVANTS : la science infuse. Puits de science. Pour être savant, il ne faut que de la mémoire. Les blaguer.»

«SCIENCE : par rapport à la religion: "un peu de Science en écarte, beaucoup y ramène" "

6 (On reviendra d'ailleurs plus bas sur les rapports entre science et religion). Sait-on aussi que nous devons à Flaubert l'une des plus savoureuses versions de l'anecdote si connue de "l'âge du capitaine ", qui concentre à l'extrême la représentation mythique, à la fois dédaigneuse et effarouchée, que le commun se fait des mathématiques? C'est Stella 
Baruk, qui dans l'un de ses ouvrages sur l'enseignement des mathématiques intitulé justement L'âge du capitaine, a retrouvé cette lettre du jeune Gustave, souffrant « de quoi se faire crever" à "barbouiller du papier avec des chiffres" lorsqu'il prépare son baccalauréat, lettre adressée à sa sœur Caroline, le 15 mars 1843 :

Je vais te donner un problème : un navire est en mer, il est parti de Boston, chargé d'indigo, il jauge deux cents tonneaux, fait voile vers Le Havre, le grand mât est cassé, il y a un mousse sur le gaillard d'avant, les passagers sont au nombre de douze, le vent souffle N.E.E. [sic], l'horloge marque trois heures un quart d'après-midi, on est au mois de mai... On demande l'âge du capitaine ${ }^{7}$.

7 J'ai d'ailleurs longtemps cru que Flaubert avait réellement inventé cette sotie, avant que d'en entendre une version rare et sensée - je veux dire, admettant une réponse logique, quoique non mathématique - et dont l'occultation par Flaubert ne rend que plus significative la perte de sens induite par la terreur mathématique ${ }^{8}$.

8 Mais c'est surtout Bouvard et Pécuchet qu'il nous faut lire et relire. On y trouve celle des figures de la science qui est le plus constamment refoulée : la bêtise. Car la science aussi, comment en irait-il autrement, a sa folie et sa bêtise. Sa folie, elle l'assume assez bien, s'accommodant des mythiques «savants fous » de la BD ou de la SF, voire revendiquant les productifs délires de certains de ses héros. Mais sa bêtise, elle veut l'ignorer. Comment nier pourtant qu'il y a une bêtise nécessaire et constitutive de la science - cet aveuglement de qui suit son seul sillon sans se laisser distraire. Ces œillères bovines, Bouvard (le bien nommé) et Pécuchet les portent, et même s'ils n'arrivent jamais au bout de leurs labours successifs, ils ont en tout cas la myope détermination de ceux qui retournent la glèbe du monde avec une science plus efficace ${ }^{9}$.

9 Ce n'est pas à cause de leur bêtise, d'ailleurs souvent moins ridicule qu'émouvante dans son ingénuité, que Bouvard et Pécuchet échouent tour à tour sur tous les continents du savoir, mais bien parce que leur désir de savoir reste trop vaste et se condamne à un papillonnage velléitaire, faute d'être assez obtus et de se faire une raison, en restreignant son objet. D'ailleurs, ce qui distingue très souvent les amateurs farfelus, auteurs de théories scientifiques hétérodoxes des chercheurs professionnels, est justement l'ampleur des vastes fresques synthétiques proposées par les premiers, à l'inverse des prudentes contributions étroitement spécialisées des seconds ${ }^{10}$. En tout cas, Flaubert garde une évidente tendresse pour ses "deux bonshommes» et leurs échecs, à l'opposé de la véritable haine qu'il voue dans Madame Bovary au pharmacien Homais, figure de la bêtise scientifique triomphante. Comme l'écrit Sartre :

[...] ce qu'il [Flaubert] reproche à Homais, c'est de se complaire à écraser sous l'entassement de petites vérités précises et coupantes les grandes inquiétudes de l'humanité. Cette bêtise invincible et victorieuse, dont les entreprises habilement menées réussissent toujours et qui, finalement, rend compte de tout le réel, de tout ce que nous sommes, il faut, pour découvrir sa hideur, son abjecte suffisance, son matérialisme à courte vue, se placer au point de vue de ce qui aurait dû être et n'a pas été, au point de vue de l'absence, du Néant, du vide, de notre vain désir et de notre délaissement. Et finalement, quelle est cette pensée caricaturale que Flaubert a logée en Homais? Eh bien, c'est tout simplement le rationalisme expérimental du docteur Flaubert ; c'est la Science tout entière, rabaissée jusqu'à l'imbécillité ${ }^{11}$.

Qui oserait dire aujourd'hui que la science - pas « tout entière ", heureusement - échappe à ce reproche et qu'elle peut se dispenser d'être attentive à ces critiques? Dans un roman beaucoup moins connu de Jacques Audiberti, Le retour du divin, on trouve un personnage féminin de « chercheuse » ou plutôt, selon les termes de l'auteur, de «savante », fort peu 
crédible quant aux conditions sociales de son travail (elle est censée travailler seule dans un laboratoire personnel perdu au cœur des Pyrénées ...), mais dont les traits personnels et la psychologie professionnelle sont remarquablement décrits :

Elle ne comprenait guère qu'on pût ne pas comprendre. Les chiffres, modelés par l'espace qui les entoure, sont clairs. À la différence des mots, ils ne fument pas, ils ne sentent pas. Les lois ne forcent personne, n'exigent rien. Elles donnent, tranquillement, la mesure $d u$ monde. Jamais impératrices furent moins contestables. À tout instant, n'importe où, dans le passé, dans l'avenir, sur la terre, aussi bien que dans la lune, les cas d'égalité des triangles rectangles jouaient sans relâche, sans fatigue, sans dégoût. Les points de suspension après les dix millièmes $\mathrm{du}$ nombre pi, pas plus que le signe de l'infini, comme un huit horizontal, ni cette marge d'approximation que se consent le calcul infinitésimal, Martine n'en tirait aucun aliment pour une rêverie dubitative, ni même pour une culture personnelle, individuelle, solitaire, incontrôlable ${ }^{12}$.

11 Nous, scientifiques, sommes trop peu nombreux, de fait, à « comprendre qu'on peut ne pas comprendre ", et à penser que la science nous permet quand même, jusque dans sa pratique, une «culture personnelle, individuelle, solitaire, incontrôlable». Et beaucoup moins nombreux encore à considérer que c'est justement cette culture (scientifique) personnelle, etc. que nous devrions faire effort pour partager, et pas seulement nos simples connaissances.

D'autres romans ont donné de la figure du scientifique des images d'une grande justesse, et qui sapent avec efficacité le mythe du savant génial et solitaire, au profit d'une représentation beaucoup plus fidèle du chercheur moderne et moyen. Ainsi Daniel Schiff, dans La ligne de Sceau, a décrit avec finesse et humour les pensées intimes d'un jeune physicien qui, tous les matins, prend le métro à Paris pour rejoindre son laboratoire à Orsay, et dont l'imagination va et vient entre les difficultés de ses équations qui l'obsèdent et les genoux de sa voisine qui ne l'obsèdent pas moins. Quant à Daniele Del Giudice, dans Atlas occidental, il met en scène avec talent la confrontation à Genève d'un vieil écrivain américain et d'un jeune physicien italien qui travaille au CERN (Centre européen de recherches nucléaires); ce roman offre une saisissante description des colossaux appareillages de la physique des particules et du rêve démiurgique de ses praticiens.

13 Pour autant, les grands hommes de la science n'en existent pas moins. Mais leurs biographies relèvent trop souvent de l'hagiographie, et ces arbres ont pour fonction essentielle de cacher la forêt. Pour sortir de l'apologétique, il faut échapper au genre trop convenu des "vies de savant" (rappelons le très savoureux pastiche, ironique et charmant, donné par Gisèle Prassinos avec son portrait de Berge Bergsky dans Brelin-leProu). Soit le cas Oppenheimer. On sait comment ce physicien des plus brillants (un bon exemple, incidemment, de scientifique dont la trop fine et trop critique intelligence aura empêché le grand talent d'aller jusqu'au génie créateur - il lui manquait un grain de bêtise...), après avoir assumé la direction scientifique du projet Manhattan (la mise au point des premières armes nucléaires à Los Alamos pendant la Seconde Guerre mondiale), fut écarté de ses responsabilités après avoir eu maille à partir avec la CIA et avoir été traduit en justice par la Commission des activités antiaméricaines en pleine hystérie maccarthyste. On connaît moins l'histoire ambiguë de ses relations avec la CIA et de ses compromissions initiales : pour accéder à son poste, il avait donné des gages et dénoncé l'un de ses amis, intellectuel américain procommuniste avant-guerre. L'alliance chez Oppenheimer d'une puissante intelligence et d'une fragile moralité a été 
remarquablement décrite par sa victime, Haakon Chevalier, dans un roman, L'homme qui voulut être Dieu - véritable tragédie antique où c'est bien l'hybris du héros qui le conduit à sa perte. Rarement le mixte d'arrogante présomption et de veule naïveté qui caractérise tant de grands noms de la science aura été si bien mis en scène. Dans cette perspective, on peut cependant citer une extraordinaire nouvelle, à la fois onirique et ironique, de Stig Dagerman, Dieu rend visite à Newton, d'une noirceur assez désespérée - mais parfaitement adéquate à l'antipathie admirative qu'on ne peut manquer d'éprouver pour le personnage de Newton.

\section{La cornue}

Encore nous faut-il comprendre ce qu'est la science. Dans cette interrogation sur la nature, la valeur et le statut de l'activité scientifique qui fait l'ordinaire de l'épistémologie, ne négligeons pas l'aide, extraordinaire, que peut nous fournir la littérature. Cette essence de la science que nous cherchons à isoler, la littérature parfois l'atteint par une distillation plus subtile que les lourdes analyses de la philosophie des sciences - cornue plus efficace qu'une trieuse mécanique.

Notre époque a vu se forger, au-delà du rationalisme scientiste, une conception plus fine, relative et contextuelle, de ce qu'est la connaissance scientifique. De Duhem à Feyerabend et Foucault, en passant par Popper et Lakatos (et même Bachelard, mais il faudrait le montrer, contre l'opinion commune ...), c'est un assouplissement progressif des critères de scientificité et des normes de rationalité qui s'est imposé1 ${ }^{13}$. Il n'en est que plus remarquable de trouver dans la littérature des intuitions fulgurantes et des énoncés percutants de ce mouvement.

Commençons par un exemple princeps. La culture du XIXe siècle a été entièrement polarisée par l'opposition entre la poussée scientiste (positiviste, moderniste, naturaliste) et les résistances (romantique, symboliste) qu'elle suscitait. À cet écartèlement, peu ont échappé. Une exception majeure : Hugo, qui, superbement, conjugue les deux courants. En témoigne ce texte magnifique, L'Art et la Science, initialement le chapitre III du William Shakespeare que Hugo écrit comme préface aux traductions du théâtre de Shakespeare par son fils François-Victor. On y trouve développée une comparaison entre l'art et la science qui n'est pas, quant au fond, d'une originalité foudroyante, puisque Hugo y affirme le caractère absolu de l'art et relatif de la science, et considère que le premier ne connait pas l'idée de progrès alors qu'elle caractérise la seconde. Mais la force de l'écriture hugolienne arrache ces énoncés à la banalité. Et surtout, pour ce qui nous concerne, Hugo montre une conception du progrès scientifique très en avance sur le positivisme de son temps. Ce progrès, pour Hugo, n'est ni linéaire, ni quantitatif, ni cumulatif. Mais laissonslui la parole :

La science est autre.

Le relatif, qui la gouverne, s'y imprime; et cette série d'empreintes du relatif, de plus en plus ressemblantes au réel, constitue la certitude mobile de l'homme.

En science, des choses ont été chefs-d'œuvre et ne le sont plus. La machine de Marly a été chef-d'œuvre.

La science cherche le mouvement perpétuel. Elle l'a trouvé ; c'est elle-même.

La science est continuellement mouvante dans son bienfait.

Tout remue en elle, tout change, tout fait peau neuve. Tout nie tout, tout détruit tout, tout crée tout, tout remplace tout. Ce qu'on acceptait hier est remis à la meule aujourd'hui. La colossale machine Science ne se repose jamais; elle n'est jamais 
satisfaite ; elle est insatiable du mieux, que l'absolu ignore. La vaccine fait question, le paratonnerre fait question. Jenner a peut-être erré, Franklin s'est peut-être trompé; cherchons encore. Cette agitation est superbe. La science est inquiète autour de l'homme; elle a ses raisons. La science fait dans le progrès le rôle d'utilité. Vénérons cette servante magnifique ${ }^{14}$.

Comment mieux dire? Hugo montre d'ailleurs dans ce texte une époustouflante connaissance de l'histoire des sciences, dont les sources mériteraient une étude détaillée ; sans doute la fréquentation d'Arago a-t-elle joué un rôle important ${ }^{15}$. Ainsi ce passage qui à lui seul montre comment chez Hugo s'articulent savoir académique et inspiration poétique, le premier retenant la seconde de basculer dans la pomposité, la seconde permettant au premier d'échapper au pédantisme :

Tout ce long tâtonnement, c'est la science. Cuvier se trompait hier, Lagrange avanthier, Leibniz avant Lagrange, Gassendi avant Leibniz, Cardan avant Gassendi, Corneille Agrippa avant Cardan, Averroès avant Agrippa, Plotin avant Averroès, Artémidore Daldien avant Plotin, Posidonius avant Artémidore, Démocrite avant Posidonius, Empédocle avant Démocrite, Carnéade avant Empédocle, Platon avant Carnéade, Phérécyde avant Platon, Pittacos avant Phérécyde, Thalès avant Pittacos, et avant Thalès Zoroastre, et avant Zoroastre Sanchoniathon, et avant Sanchoniathon Hermès, Hermès, qui signifie science, comme Orphée signifie art. oh ! l'admirable merveille que ce monceau fourmillant de rêves engendrant le réel! 0 erreurs sacrées, mères lentes, aveugles et saintes de la vérité ${ }^{16}$ !

C'est tout un cours d'épistémologie que l'on pourrait - que l'on devrait - bâtir sur ce texte de Hugo. Dans un roman de Serge Bramly, Le piège à lumière, publié avec trop peu d'échos en 1979, on trouve une réflexion des plus subtiles sur cette idée, au fond fort étrange si l'on veut bien y penser, des "lois de la nature" - idée qui caractérise la science occidentale (à la différence, par exemple, de la tradition scientifique chinoise, comme Needham l'a bien montré). Dans le livre de Bramly, une sorte de vieil illuminé a trouvé un manuscrit du XVIII siècle, décrivant en détail un château. Il veut en réaliser une maquette et embauche le narrateur pour l'assister dans sa tâche. Un mystère plane sur cette reconstruction qui finit par converger vers le cabinet de curiosités situé dans une tour du château. La description du cabinet y met en évidence un appareillage optique perfectionné - lentilles, miroirs, etc. Et voici comment les protagonistes finissent par comprendre le but de leur prédécesseur, le constructeur du château :

[...] la lumière était pour lui un symbole d'évidence, de vérité, sans doute se passionnait-il pour ses jeux à la surface des bassins du parc, dans le feuillage des arbres, sur le brillant des armures, il considérait les reflets, s'attachait aux ombres, s'ingéniait à retrouver leurs sources, à force d'observations patientes, à prévoir leur emplacement, leur éclat, à prédire leur densité. Ainsi en arrivera-t-il sans doute, peu à peu, à s'interroger sur l'infaillibilité présumée de la nature. Elle était trop à son aise dans son élément, ses manifestations y étaient trop diffuses, trop complexes pour qu'il osât attaquer chez elle; il se retira alors dans sa tour (Natura rerum magis se prodit per vexationes artis quam in libertate propria, cite-t-il), afin de la mieux cerner dans l'espace d'un laboratoire ${ }^{17}$.

19 C'est un piège qu'il s'agit de tendre à la lumière, pour la prendre en défaut. Car il n'est pas de lois sans exceptions ! Et la lumière, si elle est censée ne pas ignorer la loi, ne saurait y obéir toujours. Aussi les héros du livre reconstruisent-ils le "piège à la lumière » et se mettent-ils à l'affût: «La lumière se comporte avec une telle assurance. Pareille à ces géants immémoriaux qui se croyaient invincibles, elle s'avance à visage découvert. Elle est en fait l'esclave de ses habitudes. » Alors il table sur l'effet de surprise, sur une possible distraction. 
Exploitons sa suffisance, [dit-il]; nous avons créé ce piège, l'avons créé de toutes pièces, l'avons pourvu de ramifications infinies et changeantes: les imprévisibles circuits où nous la forçons, la prendrons bien un jour au dépourvu - il suffit d'une faute ! - ils la décontenanceront, elle trébuchera, une étourderie, nous lui ferons perdre pied une fois au moins. Commençons par endormir sa méfiance; contentons-nous, pour le moment, de problèmes aisés, d'amicales promenades, elle faillira, m'assure-t-il, vous verrez, lorsque se mettront réellement en branle les impénétrables rouages de notre machine, lorsque nous l'entraînerons dans nos dédales les plus secrets ${ }^{18}$.

20 Il y a dans cette trame romanesque une remarquable intuition de l'homologie entre recherche scientifique et enquête policière. Le chercheur, comme le détective, s'intéresse plus aux infractions qu'au respect des lois! La routine, policière comme scientifique, est celle de la vérification : papiers en règle, formules satisfaites. Mais le grand jeu est celui de la poursuite des hors-la-loi. Contrairement à ce qu'une conception frileuse de la science laisse trop souvent croire aux profanes, il n'est pas de plus grande excitation pour le chercheur que de découvrir une exception aux lois admises, une limite de validité des théories acceptées. Le jour où la théorie de la relativité einsteinienne montrera une faille ne sera pas temps de deuil mais de gloire pour le scientiflic dont le piège à lumière se sera refermé sur un photon délinquant. La fiction de Bramly met admirablement en lumière cette traque de l'illégalité qui sous-tend la recherche scientifique. En soulignant la contingence des lois de la nature, comme celle des lois de la société, elle nous aide aussi à comprendre la violence que celles-là comme celles-ci exercent. Le mouvement de rébellion que peuvent inspirer les contraintes des lois naturelles mérite d'être mieux pris en compte par les producteurs et les médiateurs de la science dans leurs relations avec les profanes, et devrait les obliger à s'interroger sur la signification profonde de la forme juridique, normative et légiférante que revêt la connaissance scientifique.

Concluons cette brève introduction à un usage épistémologique de la littérature par une incursion chez Brecht. S'il est un écrivain qui en notre siècle a entretenu avec la science un rapport profond, constitutif de toute une partie de son œuvre et révélateur de ses contradictions (qu'en bon dialecticien, il assumait par avance), c'est bien lui. De très nombreuses références à la méthode, l'esprit ou la pratique scientifique, émaillent ses essais (par exemple Le petit organon ou L'achat du cuivre), ses poèmes, son Journal de travail, ses récits (Histoires d'almanach, Dialogues d'exilés) et son théâtre (Turandot, Homme pour homme, etc.), pour aboutir à fournir la matière essentielle de sa dernière œuvre majeure, La vie de Galilée - sur laquelle nous reviendrons plus bas. Nous nous contenterons ici de l'un des aphorismes de Me-Ti ou le Livre des retournements, où Brecht se montre un précurseur de la salubre épistémologie anarchiste, ou mieux : dadaïste, selon Feyerabend (Contre la Méthode) - avec peut-être même un degré de liberté supplémentaire dans l'ironie dialectique : "On n'aurait aucune peine et on aurait grand avantage à représenter la science comme un effort pour découvrir le caractère non scientifique des affirmations et des méthodes scientifiques ${ }^{19}$ ». On se rappellera alors sans étonnement que Feyerabend a, de fait, été un temps, en sa prime jeunesse, féru de théâtre et a pu approcher Bertolt $B r e c h t^{20}$. Voilà un cas au moins où l'influence de la littérature sur l'épistémologie est directe et avérée... 


\section{La pierre de touche} questionnements et les affrontements toujours plus aigus que son rôle croissant dans la cité exige. Ne devons-nous pas, alors, prêter la plus extrême attention à ces textes où romanciers ou dramaturges ont déjà mis en page ou en scène ces débats, ces conflits? Ils nous offrent un terrain d'expérimentation, une épreuve de vérité ou de validité pour nos arguments - une pierre de touche avec laquelle tester la force et la pertinence de nos idées.

Ainsi du débat récurrent sur les relations entre science et religion et de ses modernes avatars. L'idée reçue déjà épinglée par Flaubert («un peu de Science écarte [de la religion], beaucoup y ramène ») ne cesse de revenir à la mode - un livre à succès signé en 1991 par un académicien catholique et deux médiaticiens cathodiques l'a démontré. Il est évidemment plus intelligent pour les courants spiritualistes, plutôt que de s'opposer à la science et de la dénigrer, de s'essayer à la récupérer. L'inévitable confusion épistémologique qui entoure l'émergence de nouvelles conceptions scientifiques fournit un bouillon de culture assez trouble pour tenter d'en nourrir les visions du monde les plus diverses. Ainsi, dans les premières décennies de ce siècle, c'était sur le prétendu indéterminisme quantique que l'on tentait de fonder le libre arbitre humain - on allait le chercher jusqu'au cœur de l'électron. Aujourd'hui, c'est la cosmologie et l'ambigu "principe anthropique» (selon lequel l'Univers, la forme de ses lois, la valeur de ses constantes fondamentales, est très précisément ajusté pour permettre l'apparition d'une intelligence humaine capable de le comprendre), qui sont sollicités à des fins apologétiques religieuses -ou, parfois, les formulations les plus brutales d'un évolutionnisme biologique dont la téléologie rejoint la théologie (suivant une voie naguère illustrée par Teilhard de Chardin).

Face à cette exploitation empressée, le rappel de la nécessaire prudence méthodologique, l'affirmation de l'indispensable séparation des genres entre science et religion, la référence à la laïcité de la recherche semblent bien peu efficaces. La critique rationaliste, acculée par définition à la défensive, a toutes les apparences d'une tâche à la Sisyphe. Une autre stratégie cependant est possible dans ce débat d'idées : plutôt que d'affronter de face la lourde alliance (pas si nouvelle) du spiritualisme et du scientisme, il s'agit de la prendre à revers. Cette voie, c'est un roman qui nous l'ouvre avec virtuosité et intelligence. Un roman trop peu remarqué de John Updike, Ce que pensait Roger (étrange et maladroit titre français ; le titre anglais original est Roger's Version), a pour narrateur un professeur de théologie d'une université américaine de Nouvelle-Angleterre. Âgé d'une cinquantaine d'années, il a quitté le sacerdoce pour l'enseignement et la recherche (sur les hérésies chrétiennes des premiers siècles - passionnantes au demeurant). Le voici confronté à un jeune informaticien, Dale Kohler, qui tente de convaincre le département de théologie de s'intéresser à la science contemporaine et souhaite y préparer une thèse, en invoquant pêle-mêle toutes les avancées de la science contemporaine (remarquablement présentées, il faut le dire, par Updike) :

[...] l'exposé de son projet: "Démontrer à partir des données physiques et biologiques existantes, au moyen de modèles et manipulations sur ordinateur digital électronique, l'existence de Dieu, c'est-à-dire d'une intelligence agissante et souveraine derrière tous phénomènes ${ }^{21}$ "

Cahiers de Narratologie, 18 | 2010 
25 passe sur les dimensions affective et sexuelle des rapports entre Dale et Roger via la femme et la nièce du second, dont Updike tire une matière romanesque assez épicée. Le nœud du roman reste l'affrontement métaphysique et épistémologique des deux hommes dont voici le moment crucial :

Et où se placent les fossiles précambriens ? poursuivit Dale. Voilà que brusquement des animauxmulticellulaires, il y en a partout, on dénombre sept souches, et environ cinq cents espèces - arthropodes, brachiopodes, éponges, vers. Presque tout, en fait, sauf ce que l'on aurait pu attendre - les protozoaires. Comment les cellules ont-elles appris à s'agglutiner? Et puis, au fait, comment les cellules procaryotiques, ce qu'étaient précisément les algues bleu-vert, se sont-elles transformées pour devenir nos actuelles cellules eucaryotiques, non seulement pourvues d'un nucléus mais de mitochondries, de nucléoles, de l'appareil de Golgi, et d'autres trucs dont personne n'a encore découvert la fonction. Entre les deux espèces de cellules il y a autant de différences qu'entre une chaumière et une cathédrale. - Ma foi, dis-je, en tout cas il s'est passé quelque chose, mais, pour ma part, je ne jugerais pas que j'y vois le doigt de Dieu. Tous ces raisonnements à rebours à partir des conditions actuelles pour conclure qu'elles sont hautement improbables, est-ce que vraiment ça nous donne une telle longueur d'avance sur l'homme des cavernes, qui ne comprenait pas pourquoi chaque mois la lune changeait de forme dans le ciel et en conséquence inventait un tas d'histoires sur les dieux, les blagues et les cabrioles auxquelles ils se livraient là-haut? Vous vous imaginez, dirait-on, que par obligeance Dieu est disposé à se précipiter pour combler le vide, la moindre lacune de la science. Le savant moderne n'a pas la prétention de tout savoir, il prétend uniquement savoir plus de choses que ses prédécesseurs, et aussi que les explications naturalistes paraissent se vérifier. Impossible d'avoir tout le bénéfice de la science moderne et, en même temps, de s'accrocher à la cosmologie de l'homme des cavernes. Vous gardez Dieu prisonnier de l'ignorance humaine; selon moi, Kohler, il y a trop longtemps qu'il en est prisonnier ${ }^{22}$.

On voit la subtilité et l'efficacité de l'argumentation: il est beaucoup plus pertinent de réfuter la prise en otage de la science par la religion à partir de la seconde que de la première ! Et quand Roger ajoute :

Mais ma foi, dérisoire ou non, me pousse à m'insurger avec horreur contre votre tentative, votre grossière tentative, ai-je failli dire, pour réduire Dieu au statut de fait, un fait parmi tant d'autres, pour L'induire! J'ai l'absolue conviction que mon Dieu à moi, que le vrai Dieu de n'importe qui, ne sera pas induit, ne sera jamais tributaire de statistiques, de fragments d'ossements desséchés et de vagues lueurs au bout d'un télescope ${ }^{23}$ !

et qu'il cite enfin le théologien Karl Barth ( $Q$ Quel genre de Dieu est-il, ce Dieu qu'il est besoin de démontrer $\left.{ }^{24} ? »\right)$, toute critique étroitement rationaliste devient caduque et dérisoire. Il est remarquable que ce soit une œuvre de fiction et non un essai qui intervienne ainsi avec tant de pertinence dans ce vieux débat. Rappelons au passage que l'intérêt de John Updike pour la science est loin d'être contingent, puisqu'on lui doit, dès le début de son œuvre, quelques nouvelles irrésistibles où la faune d'un étang, ou encore un groupe de dinosaures, lui servent à décrire les mœurs de la classe moyenne américaine ${ }^{25}$, de très remarquables poèmes, assez lucréciens d'inspiration et, là encore, d'une parfaite justesse scientifique, sur des thèmes physiques ${ }^{26}$.

28 Si les rapports entre science et religion posent problème, que dire des rapports entre science et politique, aujourd'hui cruciaux pour l'évolution de nos sociétés - sinon qu'il est urgent d'en mettre à l'épreuve nos conceptions. C'est Brecht ici qui nous tend une précise pierre de touche avec La vie de Galilée. Cette pièce tient une place majeure dans son œuvre. 
Elle a connu pas moins de trois versions, la première dans les années trente, où le théoricien du «théâtre de l'ère scientifique » utilise clairement la référence à la science comme fondement méthodologique : la science lui offre un modèle à la fois de rationalité et d'efficacité ; le scientifique lui apparaît comme une figure idéale de l'intellectuel, à la fois produisant un discours vrai et menant une action juste. Au mépris de sa théorie de la distanciation, Brecht fera de Galilée dans cette première version un héros essentiellement positif, combattant pour la liberté du savoir et la libération des hommes. Pendant la Seconde Guerre mondiale, Brecht, exilé aux États-Unis, voulant remonter son Galilée, le modifiera profondément, sous l'influence d'abord d'une vision beaucoup plus sceptique du héros proposée par Charles Laughton qui reprenait le rôle, puis sous l'impact de la bombe atomique larguée sur Hiroshima le 5 août 1945, qui amènera Brecht à dialectiser ses conceptions quant aux rapports entre science et société, ou, plutôt, quant au rôle social du scientifique. La version finale est d'une impressionnante complexité, tout en étant - là est le génie de Brecht - d'une grande fidélité à la vérité historique de la vie et de l'œuvre de Galilée ${ }^{27}$. Je ne peux ici que renvoyer à une analyse plus détaillée ${ }^{28}$, et me contenterai de citer la grande autocritique finale de Galilée - qui, elle, ne prétend guère, bien sûr, à la véracité historique, tant il est vrai que nous parle ici, non le savant du XVII siècle, mais un chercheur du $\mathrm{XX}^{\mathrm{e}}$ :

Le combat pour rendre le ciel mesurable est gagné à cause du doute ; à cause de la foi, le combat de la ménagère romaine pour son lait sera encore et toujours perdu. La science [...] a à voir avec ces deux combats. Une humanité trébuchante dans ce brouillard nacré de superstitions et de vieux dictons millénaires, trop ignorante pour déployer pleinement ses propres forces, ne sera pas capable de déployer les forces de la nature que vous dévoilez. Pourquoi travaillez-vous? Moi, je soutiens que le seul but de la science consiste à soulager les peines de l'existence humaine. Quand des hommes de science intimidés par des hommes de pouvoir égoïstes se contentent d'amasser le savoir pour le savoir, la science peut s'en trouver mutilée, et vos nouvelles machines pourraient ne signifier que des tourments nouveaux. Vous découvrirez peut-être avec le temps tout ce qu'on peut découvrir, et votre progrès cependant ne sera qu'une progression, qui vous éloignera de l'humanité. L'abîme entre elle et vous pourrait un jour devenir si grand qu'à votre cri de joie devant quelque nouvelle conquête pourrait répondre un cri d'horreur universel ${ }^{29}$.

Comment ne pas prendre au sérieux aujourd'hui cette adjuration de Brecht-Galilée ? À sa mort, en 1956, Brecht travaillait encore sur ce thème, puisqu'il envisageait une pièce qui aurait mis en scène un physicien contemporain, tenant à la fois d'Einstein et d'Oppenheimer, et où il aurait repris et renouvelé le mythe prométhéen: en un renversement fort brechtien, Prométhée y aurait été enchaîné par les hommes pour avoir livré aux dieux, aux puissants, le secret du feu - superbe métaphore de la découverte de l'énergie nucléaire ! Par-delà son contenu explicite, l'œuvre de Brecht, dans sa dynamique même, constitue une efficace pierre de touche pour éprouver nos conceptions quant au rôle social et à la responsabilité politique des chercheurs et témoigne de l'évolution de ces conceptions au fil du siècle; le prouve l'évolution concomitante des critiques et commentaires dont elle a fait l'objet ${ }^{30}$.

La vision de Brecht, dans sa Vie de Galilée en tout cas, a pourtant sa limite dans sa conception magique, mais encore optimiste, d'un savant qui aurait pu, s'il l'avait voulu, échapper au pouvoir et mettre sa science au service des opprimés :

Moi, en tant qu'homme de science, j'avais une possibilité unique. De mon temps l'astronomie atteignait les places publiques. Dans ces conditions tout à fait particulières, la fermeté d'un homme aurait pu provoquer de grands ébranlements. Si j'avais résisté, les physiciens auraient pu développer quelque chose comme le 
serment d'Hippocrate des médecins, la promesse d'utiliser leur science uniquement pour le bien de l'humanité ! Au point où en sont les choses, le mieux que l'on puisse espérer est une lignée de nains inventifs qui loueront leurs services à n'importe quelle cause. J'ai en outre acquis la conviction, Sarti, que je n'ai jamais été vraiment en danger. Quelques années durant, j'ai même été aussi fort que les autorités et j'ai livré mon savoir aux puissants pour qu'ils en usent, n'en usent pas ou en abusent tout comme cela servait leurs intérêts. J'ai trahi ma profession. Un homme qui a fait ce que j'ai fait ne peut être toléré dans les rangs de la science ${ }^{31}$.

Cette surévaluation du rôle individuel du chercheur et de son autonomie aurait peut-être été mise en cause dans ses œuvres restées inachevées. Mais il existe une suite, et une réplique, à $\mathrm{La}$ Vie de Galilée. C'est une tragi-comédie, Les physiciens, de Friedrich Dürrenmatt - qui a revendiqué explicitement la filiation de sa pièce avec celle de Brecht. La scène, contemporaine, est située au bord du lac Léman, dans une clinique psychiatrique de luxe dirigée par la doctoresse Mathilde von Zahn, disciple réputée de Jung. Dans la clinique, trois malades qui se croient physiciens: l'un se prend pour Einstein, le second pour Newton, et le troisième pour le plus grand physicien contemporain, Möbius, d'ailleurs étrangement disparu depuis des années. Le rideau se lève au moment où l'inspecteur Voss vient enquêter sur le meurtre de son infirmière par «Einstein »-ce qui est assez curieux, puisque "Newton » avait déjà étranglé la sienne quelques mois auparavant. La doctoresse trouve cela fort gênant pour le crédit de son établissement ... Lorsque les trois malades se retrouvent seuls, la vérité (la première vérité) éclate. « Möbius » est le vrai Möbius, épouvanté par les conséquences possibles de ses découvertes, mais ne voulant, ne pouvant pas renoncer à la recherche; alors il a « fait le fou », a prétendu avoir des apparitions - le roi Salomon lui dicterait ses travaux - et s'est fait enfermer dans cet asile de fous pour retrouver la liberté de faire de la science comme il l'entend ... « Einstein » et « Newton » sont deux de ses collègues, l'un de l'Ouest, l'autre de Est, envoyés par leurs services secrets respectifs pour récupérer Möbius et le ramener au service qui de la Liberté, qui du Peuple; démasqués par leurs infirmières, ils ont dû les assassiner. Mais Möbius refuse de quitter l'asile :

MÖBIUS : C'est curieux. Vous me vantez chacun une théorie différente, mais la réalité que vous m'offrez est la même : une prison. Alors là, je préfère mon asile de fous! Il m'assure au moins de ne pas être exploité par les hommes politiques.

EINSTEIN : Il faut tout de même prendre certains risques.

MÖBIUS : Il y a des risques qu'on ne doit jamais courir, par exemple la destruction de l'humanité. Nous savons ce que le monde fait des armes qu'il possède déjà ; ce qu'il ferait de celles que mes découvertes lui fourniraient, nous pouvons l'imaginer sans peine. J'ai réglé ma conduite en conséquence. J'étais pauvre. J'avais une femme et trois enfants. La carrière universitaire c'était la gloire, l'industrie c'était la fortune. Les deux voies étaient trop dangereuses. J'aurais été obligé de publier mes travaux, ce qui aurait entraîné le bouleversement de la physique et, par contrecoup, l'effondrement de l'économie. Ma conscience me forçait à choisir une autre issue. J'ai quitté l'université, j'ai lâché l'industrie et j'ai abandonné ma famille à son sort. J'ai choisi de me cacher sous la cape du bouffon. Il a suffi de prétendre que le roi Salomon m'apparaissait, pour être embarqué dare-dare dans un asile d'aliénés.

NEWTON : Mais ce n'était pas une solution!

MÖBIUS: C'était le seul comportement raisonnable. La physique s'est heurtée aux limites du connaissable. Nous possédons quelques lois compréhensibles et certaines relations fondamentales entre des phénomènes incompréhensibles, c'est tout. Tout le reste nous échappe et demeure un mystère inaccessible à la raison. Nous sommes au bout de notre chemin. Mais l'humanité n'en est pas encore là. Nous avons mené des combats d'avant-garde, mais personne n'a suivi et nous avons abouti dans le désert. La science est devenue terrible et la recherche dangereuse. Nos 
connaissances sont mortelles. Il nous faut capituler devant le monde tel qu'il est. Nous sommes trop forts pour lui. Nous causons sa perte. Il faut lui reprendre la science que nous lui avons donnée. Moi, j'ai repris la mienne. Il n'y a pas d'autre solution, pour vous non plus.

EINSTEIN : Ce qui signifie?

MÖBIUS : Vous devez rester avec moi, parmi les fous.

NEWTON : Nous?

MÖBIUS : Tous les deux.

Silence ${ }^{32}$. pour préserver l'humanité des terrifiantes retombées de la science nouvelle. Happy end sur le thème de la conscience retrouvée et de la responsabilité assumée ? Ce serait mal connaître Dürrenmatt... La situation se retourne : au fil des années, la doctoresse von Zahn a mis la main sur les papiers du naif Möbius et fondé sur ses découvertes un gigantesque trust militaro-industriel multinational qui est en train d'accaparer la planète. En croyant s'isoler du monde et se refuser à l'exploitation de leurs travaux, nos physiciens se sont d'eux-mêmes jetés dans la gueule du loup et se retrouvent au cœur du système en train de l'alimenter. Salubre et sarcastique mise en garde : Dürrenmatt nous fournit, avec Les physiciens, l'aune à quoi mesurer la portée et l'efficacité de nos réactions qui relèvent trop souvent d'un idéalisme naïf et individuel et ne sont guère à l'échelle des enjeux du développement technoscientifique ${ }^{33}$.

Notons au passage que le thème du chercheur effrayé par ses propres découvertes et fuyant le monde pour le préserver - sans succès - a été traité par Sciascia, dans $L a$ disparition de Majorana, à partir d'un épisode réel : Ettore Majorana était un jeune et génial physicien italien qui disparut brusquement en 1936, s'étant probablement suicidé ou, peut-être, enfermé dans un couvent. Le roman-enquête de Sciascia, bien qu'assez problématique du point de vue historique (il attribue à Majorana une prescience peu plausible des conséquences de ses recherches en physique nucléaire) est, comme d'habitude, d'une lucidité impitoyable.

On pourrait longuement poursuivre cette plongée littéraire au cœur de la science: développer de profondes et fraîches considérations épistémologiques à partir de l'œuvre poétique de Francis Ponge ou, de façon plus inattendue, de certaines bandes dessinées comme celles de Masse ; revoir d'un œil neuf les grandes figures de la science au travers des poèmes que leur a consacrés Hans Magnus Enzensberger ; relire, pour les perspectives qu'ils ouvrent sur la science, bien des romans ou des nouvelles (Elsa Triolet, René Daumal, Pierre Boulle, François Weyergans et tant d'autres). Mais il ne s'agit pas ici d'une anthologie ${ }^{34}$.

\section{Quelques leçons}

L'aide que la littérature peut apporter à la science, ce n'est pas par hasard que je l'ai placée sous la triple enseigne d'instruments - le miroir, la cornue, la pierre de touche qui appartiennent plus au laboratoire de l'alchimiste qu'à celui du chercheur moderne. Car derrière la transmutation de la matière, inerte ou vivante - que réalisent si aisément aujourd'hui le physicien nucléaire ou le biologiste généticien -, c'était la transformation de l'esprit et de la conscience humaines que visaient les prédécesseurs de la science moderne, et dont on ne se risquerait pas à affirmer que leurs héritiers l'ont beaucoup fait progresser. C'est pourquoi, outre ses œuvres et ce qu'elles peuvent nous dire de la

Cahiers de Narratologie, 18 | 2010 
science, c'est à une pratique essentielle de la littérature que nous, scientifiques, serions bien inspirés de réfléchir plus avant : je veux parler de la critique. S'il va de soi qu'il existe en littérature, comme en musique ou en peinture, une activité critique explicite, spécifique et reconnue pour telle, il n'en est certes pas de même dans la science. La prétention des producteurs de science à être eux-mêmes, et seuls, maitres de son évaluation et de ses orientations, n'est pas plus acceptable en droit que la position de juge et partie en quelque autre domaine. Au surplus, les processus d'arbitrage interne à la communauté scientifique connaissent aujourd'hui une crise évidente. C'est donc d'une véritable critique de science, comme il existe une critique d'art, une critique littéraire, que nous avons besoin dorénavant ${ }^{35}$.

Ce sont d'abord des leçons de savoir-vivre, de morale et de maintien, que la littérature peut offrir à la science. Mais on ne saurait refuser au passage quelques leçons d'imagination. Si éparses et en tout cas imprévisibles soient-elles, les occasions existent où telle œuvre littéraire pourrait à point nommé suggérer la solution d'un problème scientifique. On peut au moins repérer après coup certaines prémonitions géniales - telle la poétique résolution d'une énigme cosmologique majeure, le "paradoxe de la nuit noire »: si l'univers est infini et homogène, alors dans quelque direction que nous regardions, notre regard devrait buter sur une étoile (comme celui d'un promeneur sur un tronc d'arbre lorsqu'il regarde autour de lui en pleine forêt), et le ciel devrait nous apparaître uniformément aussi brillant que le Soleil. Ce paradoxe, la science cosmologique n'a trouvé sa solution que récemment, un siècle après qu'Edgar Poe, dans Eurêka, en eut l'intuition ${ }^{36}$. Sans vouloir absurdement trouver des réponses à tous les problèmes de la science entre les lignes des romans, il n'est pas interdit de penser qu'une meilleure fréquentation de la fiction littéraire pourrait assouplir et développer l'imagination scientifique ${ }^{37}$.

Enfin, et plus sérieusement, ce sont tout simplement des leçons d'écriture que la littérature peut proposer à la science. Si les scientifiques doivent, je le crois, lire ces livres qui nous parlent de la science, et souvent avec plus de pertinence ou de vigueur que trop d'essais théoriques, ils doivent aussi lire, lire tous les livres - et d'abord pour apprendre à écrire! Ce serait encore une étude à entreprendre que d'analyser la pauvreté, le schématisme, la médiocrité de l'écriture scientifique actuelle. La rigide codification formelle qui est de règle aujourd'hui dans les publications professionnelles des chercheurs s'accompagne, on peut le montrer, d'un appauvrissement de la pensée et d'un affaiblissement de l'échange ${ }^{38}$. Cette banalisation de l'écriture a de sérieuses conséquences sur la qualité des communications entre les professionnels de la science et ses profanes. Aussi faut-il saluer comme une contribution notable au renouveau et à l'enrichissement des indispensables médiations entre le milieu scientifique et le corps social l'apparition d'un véritable souci de l'écriture, d'une exigence de la forme écrite, dans des œuvres que, du coup, on préférera appeler « de culture scientifique » plutôt que «de vulgarisation scientifique». Pour ne prendre que quelques exemples: c'est Stella Baruk montrant le poids de la langue dans la pratique et l'enseignement des mathématiques, et la faisant jouer à plein dans ses livres, jusque dans ce Dictionnaire de mathématiques élémentaires, qui est d'abord un dictionnaire de langue; c'est le botaniste Francis Hallé qui, écrivant sur les Tropiques, nourrit son texte de citations littéraires, depuis Conrad jusqu'à Duras, et aurait voulu emprunter son titre à Serge Gainsbourg : Sous le soleil, exactement ...; c'est le mathématicien Ivar Ekeland construisant entièrement son livre $A u$ hasard sur un profond enracinement dans le texte et l'esprit des sagas 
scandinaves; c'est le biologiste Jacques Ninio, alternant dans La biologie buissonnière, développements sur les découvertes récentes de sa science et témoignages personnels en forme de petites nouvelles. Et l'on peut même trouver de véritables romans sur les recherches contemporaines, écrits par des écrivains, en collaboration avec des scientifiques - et illustrés ${ }^{39}$. Il n'est pas jusqu'à l'histoire des sciences qui ne s'essaie à la fiction: dans Les pensées nocturnes d'un physicien classique, c'est sous forme romanesque que l'historien Russell Mac Cormmack, synthétisant les recherches académiques, retrace les bouleversements de la physique au début de ce siècle. Ce recours (ce retour ?) à la littérature, on peut le voir à l'œuvre aussi dans le mouvement de rénovation des grands établissements de culture scientifique et technique. Coup sur coup, le Muséum d'Histoire Naturelle et le Musée National des Techniques ont éprouvé le besoin d'enraciner leur image et de ressourcer leur esprit au travers de petites anthologies littéraires, recueil des visions et des émotions qu'ils ont pu inspirer ${ }^{40}$. Il faudra revenir sur l'écrit de la science ... Nous, scientifiques, sommes trop seuls. On nous invite parfois à sortir de nos laboratoires et à présenter au monde nos trouvailles. Mais nous sommes si mal élevés, si gauches que, souvent, notre maladresse ennuie et notre brutalité effraie la société. Aussi avons-nous besoin que l'on s'occupe de nous et de notre science, que l'on vienne vers nous, nous aider, nous policer. Dürrenmatt, justement, l'avait compris; dans Albert Einstein, texte d'une ironique et salutaire méditation sur Einstein et Spinoza, conférence faite à l'invitation de l'Ecole polytechnique de Zurich, il commence ainsi son adresse aux étudiants et chercheurs scientifiques réunis pour l'écouter :

Si j'ai accepté l'invitation de l'Ecole polytechnique fédérale de donner une conférence sur Einstein, c'est pour la raison suivante: à l'heure actuelle, les mathématiques, les sciences physiques et naturelles et la philosophie sont à tel point interdépendantes que le profane doit, lui aussi, s'atteler à trancher ce nœud gordien. Car, abandonner physiciens, mathématiciens et philosophes à eux-mêmes, c'est les refouler définitivement dans le ghetto de leur spécialité où, oubliés et déconcertés, ils sont à la merci des technocrates et des idéologues, comme ils l'ont toujours été et ne cesseront de l'être ${ }^{41}$.

Merci aux romanciers, aux dramaturges, aux poètes, de ne pas nous abandonner à nousmêmes.

\section{BIBLIOGRAPHIE}

AUDIBERTI, Jacques,

1983, Le retour du divin, Gallimard.

BARUK, Stella

1992 a, L'âge du capitaine, Seuil.

1992 b, Dictionnaire de mathématiques élémentaires, Seuil.

BOULLE, Pierre

1992, $E=M c^{2}$, Julliard 
BOUVARD, C. et RATINET, A.

1957, Nouvelle table de logarithmes, Hachette.

BRAFFORT, Paul

1995, «L'espace-temps des voyages littéraires », Alliage, n²2, p. 63-70; et surtout, Les noces de la science et de la littérature, Diderot, 1996.

BRAMLY, Serge

1979, Le piège à lumière, Flammarion.

BRECHT, Bertolt

1968, Me-Ti ou le Livre des retournements, Paris, L'Arche.

1990, La vie de Galilée, L'Arche.

CHEVALIER, Haakon

1960, L'homme qui voulut être Dieu, Seuil.

DAGERMAN, Stig

1976, Dieu rend visite à Newton, Denoël.

DAUMAL, René

1981, Le mont Analogue, Gallimard.

DÜRRENMATT, Friedrich

1988, Les physiciens, L'Âge d'Homme.

1982, Albert Einstein, L’Aire.

DEL GIUDICE, Daniele

1987, Atlas occidental, Seuil.

EKELAND, Ivar

1991, Au hasard, Seuil.

ENZENSBERGER, Hans Magnus

1987, Mausolée, Alinéa.

FEYERABEND, Paul

1979, Contre la Méthode, Seuil.

1996, Tuer le temps (une autobiographie), Seuil.

FLAUBERT, Gustave

1979, Bouvard et Pécuchet (suivi du Dictionnaire des idées reçues), Gallimard.

FRIEDMAN, Allan, et DONLEY, Carol

1985, Einstein as Myth and Muse, Cambridge University Press.

GALILEI, Galileo

1992 a, Le messager des étoiles, Seuil.

1992 b, Dialogue sur les deux grands systèmes du monde, Seuil. 
Voir aussi Alliage, n 13, automne 1992, « Dialogue avec Galilée ».

GOLDSMITH, Maurice

1986, The Science Critic, Londres, Routledge \& Keagan Paul.

1990, «Critique de science, une nouvelle profession? ", Alliage, n 3, p. 3-7.

HALLÉ, Francis

1993, Un monde sans hiver, Seuil.

HARRISON, Edward

1990, Le noir de la nuit, Seuil.

HUGO, Victor

1985, L'Art et la Science, Actes-Sud.

LEVI, Primo

1990, « La chimie et l'écriture », Alliage, n 5, p. 45-49.

LÉVY-LEBLOND, Jean-Marc

1979, « RB. et G.G., la science en scène » in Lévy-Leblond, 1984, p. 103-119.

1980 a, « La science, ou la culture ? » in Lévy-Leblond, 1984, p. 87-101.

1980 b, « Éloge des théories fausses » in Lévy-Leblond, 1984, p. 76-84.

1984, L'esprit de sel (Science, culture, politique), Paris, Le Seuil.

1986, Mettre la science en culture, Nice, Anais.

1989 a, « La science en mal de critique », Verba Volant, n 1, p. 69-77; cf. supra, p. 149.

1989 b, « Les hémisphères de Magdebourg », Alliage, nº 1, p.3-4.

2004, La science en mal de culture, Futuribles

MAC CORMMACK, Russel

1986, Les pensées nocturnes d'un physicien classique, Londreys.

MASSE, Francis

1985, Les deux du balcon, Castermann.

MERCIER, Alain

1992, Les Arts et Métiers en toutes lettres, CNAM.

NINIO, Jacques

1991, La biologie buissonnière, Seuil.

PELOT, Pierre, COPPENS, Yves, et LIBERATORE, Tanino

1990, Le rêve de Lucy, Seuil.

PONGE, Francis

1966, Le parti pris des choses, Gallimard.

1983, Nioque de l'avant-printemps, Gallimard.

PRASSINOS, Gisèle 
1975, Brelin-Le-Frou, Belfond.

PYNCHON, Thomas

1988, L'arc-en-ciel de la gravité, Seuil.

1990, « Le retour du roi Ludd?», Alliage, n 4, p. 71-80.

QUENEAU, Raymond

1937, Odile, Gallimard.

1938, Les enfants du limon, Gallimard.

RAICHVARG, Daniel

1992, « La Fille de Galilée - Dramaturgie et scientisme », Alliage, n 13, p. 49-60.

1993, Science et spectacle, figures d'une rencontre, Nice, Z’Éditions.

SARRAUTE, Nathalie

1987, L'ère du soupçon, Gallimard.

SARTRE, Jean-Paul

1971, L'Idiot de la famille, Gallimard.

SCIASCIA, Leonardo

1984, La disparition de Majorana, Flammarion.

SCHIFF, Daniel

1983, La ligne de Sceaux, Minuit.

TAQUET, Philippe

1991, Les bonnes feuilles du Jardin des Plantes, Muséum d'histoire naturelle.

TOULOTTE, Muriel

1996, « De Géo à Démos - Hugo et Arago ", Alliage, n 26,27-34.

TRIOLET, Elsa

1959, Luna Park, Gallimard.

UPDlKE, John

1975, Femmes et musées, et autres nouvelles, Gallimard.

1988 a, Ce que pensait Roger, Gallimard.

1988 b, « Sept odes à des processus naturels » in La condition naturelle, Gallimard.

WEYERGANS, François

1986, La vie d'un bébé, Gallimard.

\section{NOTES}

1. Lévy-Leblond, 1984, 1986, 2004. Les références bibliographiques, dont la nature même de ce texte explique l'abondance, sont rassemblées à la fin du chapitre.

2. Sarraute, 1956. 
3. Friedman et Donley, 1985.

4. Braffort, 1995.

5. Levi, 1990.

6. Voir aussi Pynchon, 1990.

7. In Baruk, 1992 a.

8. Voir « La langue tire la science », in La pierre de touche. La science à l'épreuve... , Paris, Gallimard Folio, 1996, pp. 249-251.

9. Rappelons après Queneau que cette quintessence des labeurs calculatoires, la table de logarithmes, dans la version qu'ont utilisée les lycéens pendant de nombreuses décennies, avait pour auteurs... MM. Bouvard et Ratinet.

10. Les marges de la science où errent tant de Quichotte de la physique, de marginaux de la cosmologie, d'enfants perdus de la biologie, attendent encore les études qu'ils méritent - et qui éclaireraient singulièrement la science "normale" (Lévy-Leblond, 1980 b). En attendant, c'est encore à la littérature, en l'occurrence au roman de Queneau, Les enfants du limon, que l'on doit la plus sérieuse reconnaissance de ces marges.

11. Sartre, 1971, p. 644.

12. Audiberti, 1983, p. 126.

13. Voir «La chauve-souris et la chouette », in La pierre de touche. La science à l'épreuve... , cit., p. 269.

14. Hugo, 1985, p. 18-19.

15. Toulotte, 1996.

16. Hugo, 1985, p. 27-28.

17. Bramly, 1979, p. 146.

18. Ibid., p. 153.

19. Brecht, 1968 , p. 79.

20. Feyerabend, 1996.

21. Updike, 1988 a, p. 98.

22. Ibid., p. 103.

23. Ibid., p. 113.

24. Ibid., p. 267.

25. Updike, 1975.

26. "Sept odes à des phénomènes naturels ", in Updike, 1988 b.

27. Seul Antoine Vitez, dans ce qui fut sa dernière mise en scène, a su en 1991 à la ComédieFrançaise rendre pleinement la richesse et la puissance de la pièce de Brecht. Il en reste heureusement une version filmée par Hugo Santiago.

28. Lévy-Leblond, 1979.

29. Brecht, 1990, p. 131.

30. Lévy-Leblond, 1979.

31. Brecht, 1990, p. 131-132.

32. Dürrenmatt, 1988, p. 85-87.

33. Pour une étude générale sur les relations entre la science et le théâtre, voir les travaux de Daniel Raichvarg (Raichvarg, 1993).

34. Le lecteur s'étonnera peut-être de ne voir citer aucun auteur de science-fiction. C'est que la $\mathrm{SF}$, littérature de genre, ne tient le plus souvent à la science que par de superficiels traits de pure forme. Mais c'est là une discussion à mener ailleurs de façon plus approfondie. Voir cependant « Hypotheses fingo ", in La pierre de touche. La science à l'épreuve... , cit., p. 219.

35. Voir Goldsmith, 1986, 1990; Lévy-Leblond, 1989 a.

36. Voir, pour l'histoire et la résolution de l'énigme, Harrison, 1990.

37. Et c'est peut-être ici que la science-fiction stricto sensu peut jouer un certain rôle (et le joue d'ailleurs déjà, sans nul doute). 
38. On ne saurait trop recommander, à titre de comparaison et pour leur intérêt littéraire propre, la lecture des œuvres de Galilée (Galilei, 1992 a et b)

39. Pelot, Coppens et Liberatore, 1990

40. Taquet, 1991 ; Mercier, 1992

41. Dürrenmatt, 1982, p. 9

\section{AUTEUR}

\section{JEAN-MARC LÉVY-LEBLOND}

Université de Nice-Sophia Antipolis 